\title{
LEVANTAMENTO EXPERIMENTAL DA ASSOCIAÇÃO EM SÉRIE DE BOMBAS CENTRÍFUGAS COM POTÊNCIA DE $1 / 2$ CV
}

\author{
BEng. Rodrigo José da Silva ${ }^{(1)}$ (js.rodrigo_@hotmail.com), BEng. Odilon Hítalo Castro Oliveira ${ }^{(2)}$ \\ (hitalo.castro@hotmail.com), Dr. Guillermo Vilalta Alonso ${ }^{(3)}$ (gvilalta@ufsj.edu.br)
}

(1) Universidade Federal de São João del Rei (UFSJ); Programa de Pós-Graduação em Engenharia Mecânica - PPMEC

(2) Universidade Federal de São João del Rei (UFSJ)

(3) Universidade Federal de São João del Rei (UFSJ); Departamento de Ciências Térmicas e dos Fluídos - DCTEF

RESUMO: Bombas centrífugas são máquinas operatrizes que conferem energia a um fluido para proporcioná-lo deslocamento. A associação de duas ou mais bombas é um processo comum para se obter demandas específicas de vazão ou carga. A associação de bombas em série proporciona a possibilidade de obter-se cargas maiores para determinadas situações. O objetivo do presente trabalho se resumiu a efetuar - levantamento experimental da curva característica que rege a relação entre carga e vazão numa associação em série de duas bombas centrifugas com potência de $1 / 2$ CV. Foi utilizada bancada disponível no Departamento de Ciências Térmicas e dos Fluídos da Universidade Federal de São João del Rei. Os dados experimentais (vazão e carga) obtidos experimentalmente foram utilizados para gerar o gráfico que representa a curva característica da associação em série que, posteriormente, foi comparada à obtida através dos dados do fabricante. O ponto de operação do sistema associado em série foi comparado com o obtido - em trabalho anterior - na associação em paralelo, com base nos parâmetros de operação para máximo rendimento fornecidos pelo fabricante.

PALAVRAS-CHAVE: BOMBAS CENTRÍFUGAS, ASSOCIAÇÃO EM SÉRIE, LEVANTAMENTO EXPERIMENTAL

\section{EXPERIMENTAL SURVEY OF SERIES ASSOCIATION OF CENTRIFUGAL PUMPS WITH POWER OF $1 \frac{1}{2}$ CV}

ABSTRACT: Centrifugal pumps are machine tools that energize a fluid to provide displacement. Combining two or more pumps is a common process for meeting specific flow or load demands. The combination of pumps in series provides the possibility to obtain higher loads for certain situations. The aim of the present paper was to perform an experimental survey of the characteristic curve governing the relationship between load and flow in a series combination of two centrifugal pumps with power of $1 / 2 \mathrm{CV}$. A workbench available at the Department of Thermal and Fluid Sciences of the Federal University of São João del Rei was used. Experimental data (flow and load) obtained experimentally were used to generate the graph that represents the characteristic curve of the series association, which subsequently was compared to that obtained from the manufacturer's data. The operating point of the series-associated system was compared to that obtained - in previous work - in the parallel association, based on the operating parameters for maximum performance provided by the manufacturer.

KEYWORDS: CENTRIFUGAL PUMPS, SERIES ASSOCIATION, EXPERIMENTAL SURVEY 


\section{$\mathrm{XIX}$ CIONEMI}

\section{INTRODUÇÃO}

“Desde quando o ser humano começou a viver em sociedade, tornou-se necessário que ele convivesse em locais próximos de algum tipo de fonte de água. Com a evolução da civilização humana, foi preciso criar algum sistema que fornece água para a população, primeiramente de uma forma arcaica, com água escoando por gravidade através de canais, até atualmente com os sistemas sofisticados de distribuição de água" (MACINTYRE, 1982). As bombas são máquinas operatrizes que fornecem energia ao fluido, a fim de deslocá-lo de um referencial a outro; são amplamente utilizadas em sistemas de bombeamento e abastecimento de água em plantas industriais, residências, no transporte de diversos tipos de fluidos.

Por requisições de projeto, o recalque de um fluido é realizado a determinadas vazão e altura manométrica; sob estas condições, realiza-se o dimensionamento do sistema, de modo que o maior rendimento possível seja obtido. A Figura 1 representa a típica curva característica de uma bomba centrífuga,

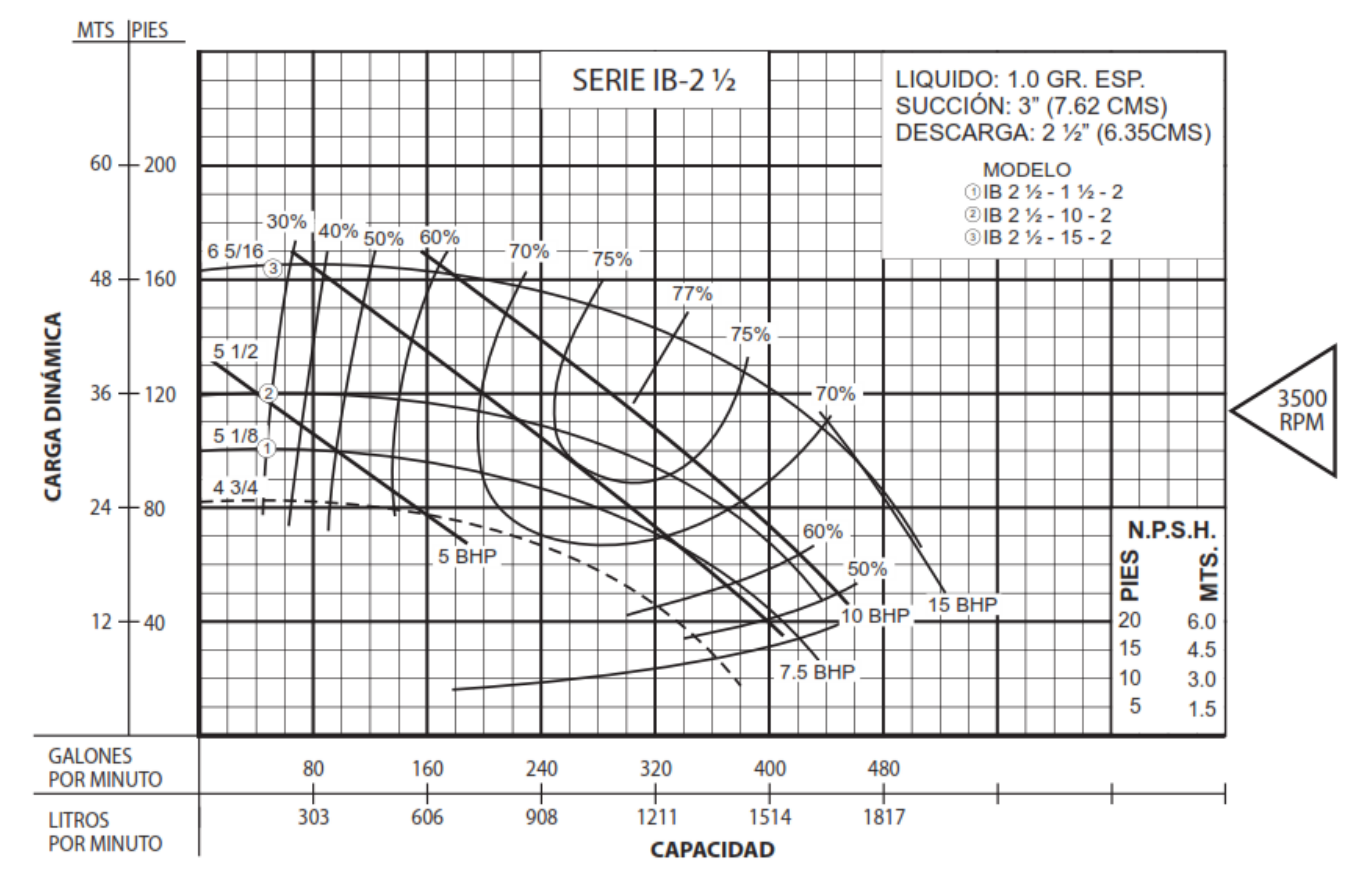

Figura 1: Curvas características de bombas centrífugas. Fonte: Hidroservicos Ambientales (2018). 


\section{$\mathbf{X I X}$ CENEMI}

tendo no eixo vertical a representação da altura manométrica e no eixo horizontal a vazão recalcada pela bomba. Curvas características de bombas centrífugas possuem uma relação polinomial de segundo grau entre carga e vazão.

Quanto à associação de bombas, dispõe-se da associação em paralelo quando se deseja aumentar a vazão de um sistema, à mesma carga; na associação em série, pelo contrário, deseja-se aumentar a carga a uma mesma vazão. A primeira configuração tem vantagem sobre a segunda em casos de manutenção, pois, um eventual problema em um sistema em paralelo não requer interrupção no funcionamento de todas as bombas; ao contrário da segunda, em série, onde o cessar de uma das bombas afeta todo o sistema de bombeamento. Uma alternativa para tal revés em associações em série, seria adotar um sistema bypass, onde o fluido contornaria a bomba defeituosa, por uma tubulação auxiliar, continuando seu percurso até a próxima bomba; todavia, isso seria feito ao custo do aumento de tubulação, válvulas e da introdução de perdas de carga no sistema. Outra desvantagem relacionada à associação em série, por si só, é a impossibilidade de efetuar controle da vazão de forma eficiente: um aumento na vazão máxima possível sem adicionar bombas em paralelo é inexecutável; e a única forma de diminuir a vazão do sistema em série seria através da introdução de perdas de carga - estrangulamento de uma válvula. Além do mais, a vazão máxima do sistema é sempre limitada à bomba cuja capacidade máxima de recalque é a menor do conjunto. Não obstante, como já bem definido, a associação em série permite flexibilidade para com a associação de bombas diferentes entre si além de um aumento na carga total entregue ao fluído.

A Figura 2 ilustra, esquematicamente, um sistema de bombas em série. A tubulação advinda da descarga de uma bomba se conecta diretamente com a sucção da bomba sequente. Nesta configuração, tem-se a mesma vazão entre todas as bombas, o que implica que a vazão em cada uma das bombas será igual à vazão total do sistema. Todavia, a carga total será igual ao somatório do incremento fornecido por cada bomba. 


\section{$\mathrm{XIX}$ \\ CIDNEMI}

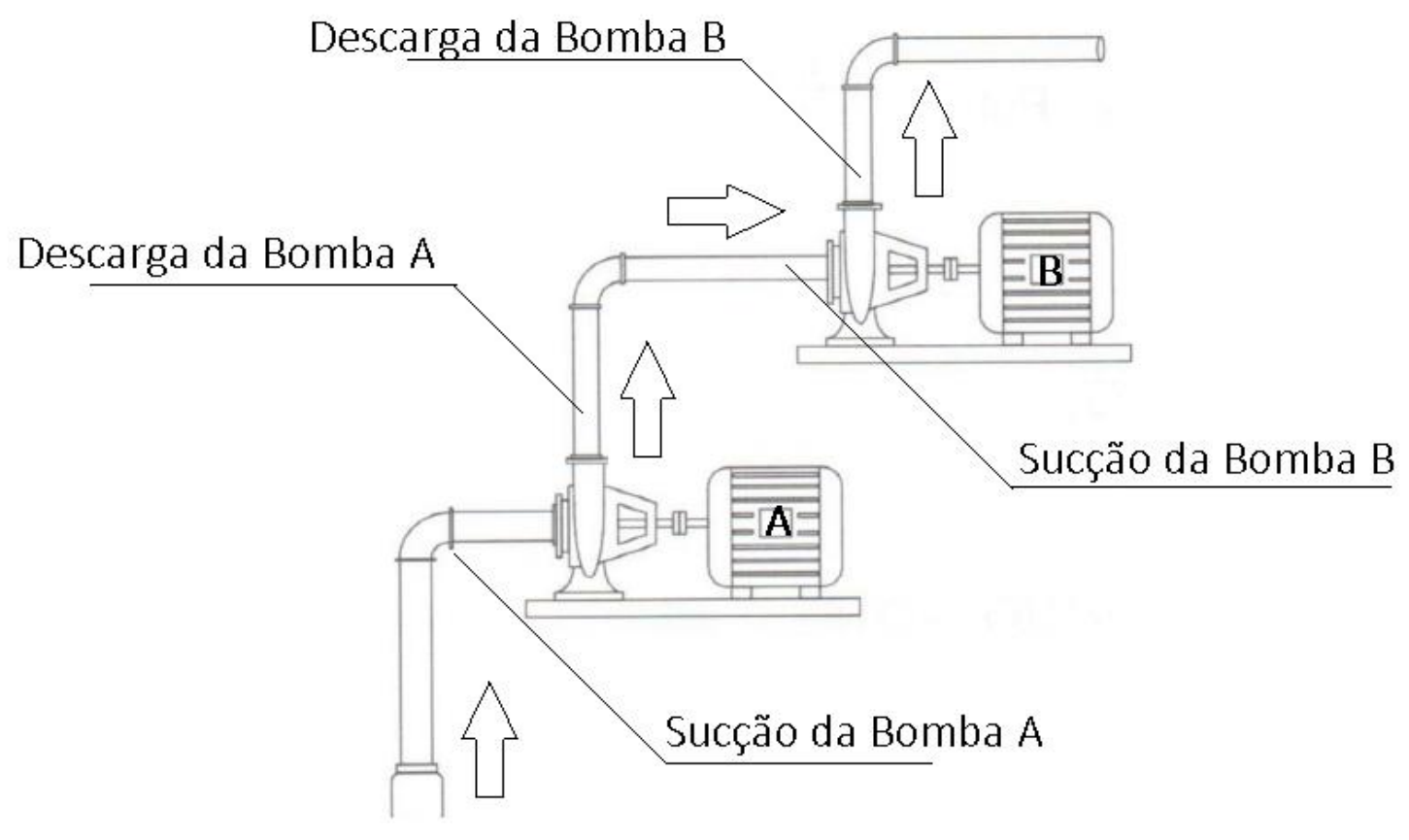

Figura 2: Associação de bombas em série. Fonte: Autores.

Uma vez que, para um mesmo valor de vazão, as cargas de cada bomba são somadas numa associação em série, a curva característica de um conjunto de duas bombas associadas dessa forma pode ser determinada somando as cargas de cada bomba correspondentes a um mesmo valor de vazão, conforme a Figura 3.

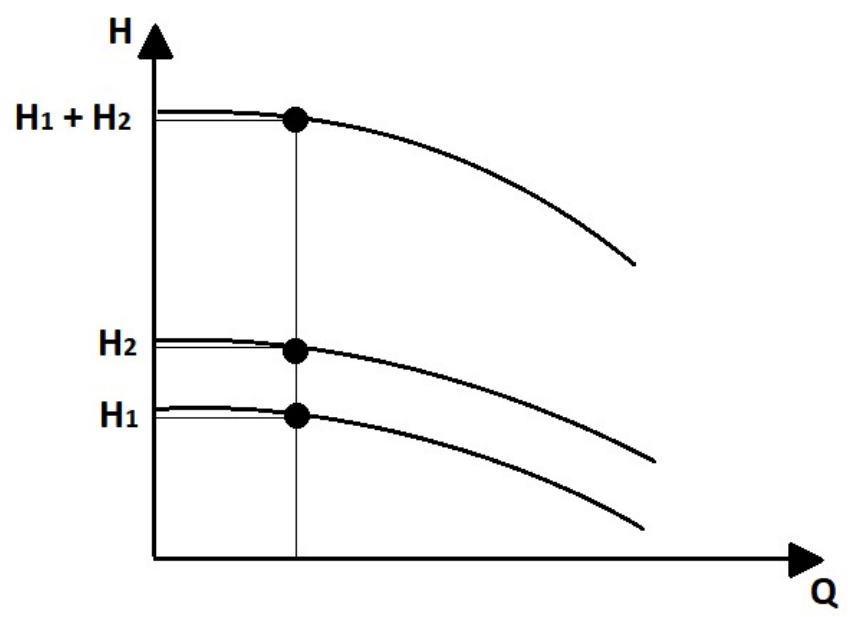

Figura 3: Curva da associação em série de duas bombas. Fonte: Autores. 


\section{$\mathbf{X I X}$ \\ CENEMI}

\subsection{Objetivos}

O presente trabalho partiu do objetivo de tornar-se um complemento do de SILVA (2018) publicado nos anais da edição do XVIII CONEMI; que tratou especificamente do levantamento da curva característica da associação em paralelo para o mesmo conjunto de bombas e a mesma bancada de testes.

A Figura 4 apresenta a curva experimental da associação em paralelo obtida por SILVA (2018). A vazão máxima obtida no ponto de operação da associação em paralelo aproximadamente $80 \mathrm{~L} / \mathrm{min}$ - foi comparada com a vazão correspondente para o máximo rendimento possível, averiguada em selo do fabricante anexado às bombas. Embora o ponto de máximo rendimento se dê, segundo Selo Procel de Economia das bombas, para uma vazão aproximada de $88 \mathrm{~L} / \mathrm{min}$ - próxima da vazão de $80 \mathrm{~L} / \mathrm{min}$ apresentada com a válvula totalmente aberta -, SILVA ressaltou que a curva na Figura 4 representa uma associação em paralelo de duas bombas idênticas e, portanto, cada uma delas opera a uma vazão aproximada de 40 L/min (bem menor que a vazão correspondente para o máximo rendimento); já que neste tipo de associação a vazão total é a soma das vazões individuais de cada bomba - diferente da associação em série, em que a vazão é única para o sistema e cada uma das bombas.

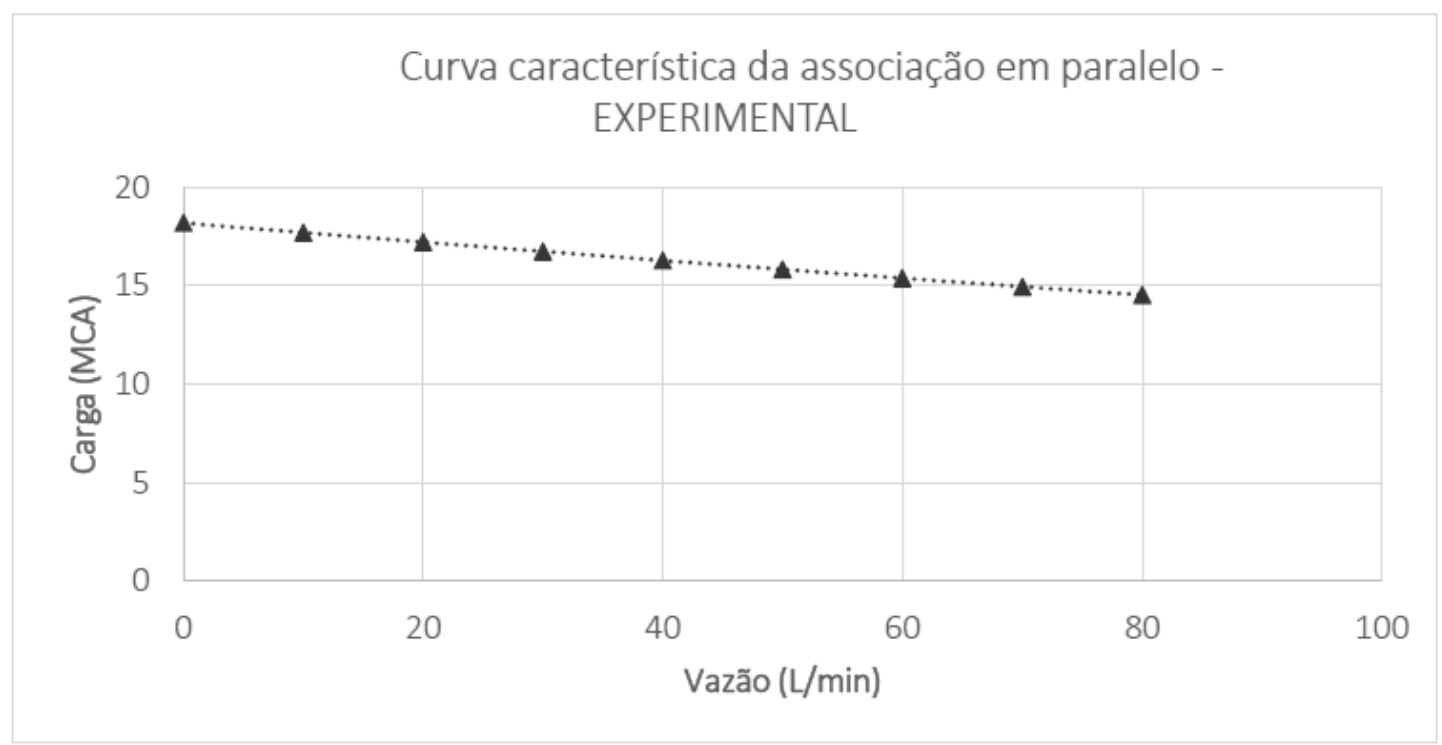

Figura 4: Curva da associação em paralelo para a bancada em estudo. Fonte: SILVA (2018). 


\section{$\mathbf{X I X}$ \\ CIDNEMI}

Por conseguinte, além do levantamento da curva para associação em série, o presente trabalho também se comprometeu a comparar o ponto de operação do sistema de bombas associados em série com os parâmetros de funcionamento para máximo rendimento fornecidos pelo fabricante. Para tanto, foi adotada metodologia similar à de SILVA (2018):

1. Aplicar a metodologia para levantamento da curva característica de carga vs. vazão de uma associação em série de duas bombas idênticas (Texius, modelo TBHWS-RN 1/2 CV);

2. Obter de forma teórica, através da curva características fornecida pelo fabricante, a curva característica para a associação em série.

3. Comparar a curva característica obtida experimentalmente com aquela obtida mediante cálculo teórico.

4. Comparar a vazão máxima da associação em série - ponto de operação - com os parâmetros de máximo rendimento fornecidos pelo fabricante.

\section{MATERIAIS E MÉTODOS}

O procedimento experimental foi realizado, assim como SILVA (2018), em uma bancada de bombas pertencente ao Departamento de Ciências Térmicas e dos Fluídos da Universidade Federal de São João del Rei (DCTEF-UFSJ). A bancada é constituída por:

- Reservatório de água, Manômetros (Sucção e Descarga), Rotâmetros e duas Bombas com as características do objetivo de estudo - Bomba Centrífuga Texius, modelo TBHWS-RN $1 / 2$ CV (Figura 5A)

- Quatro válvulas de controle (Figura 5B)

- Válvula de regulagem (Figura 5C) 


\section{XIX CIDNEMI}

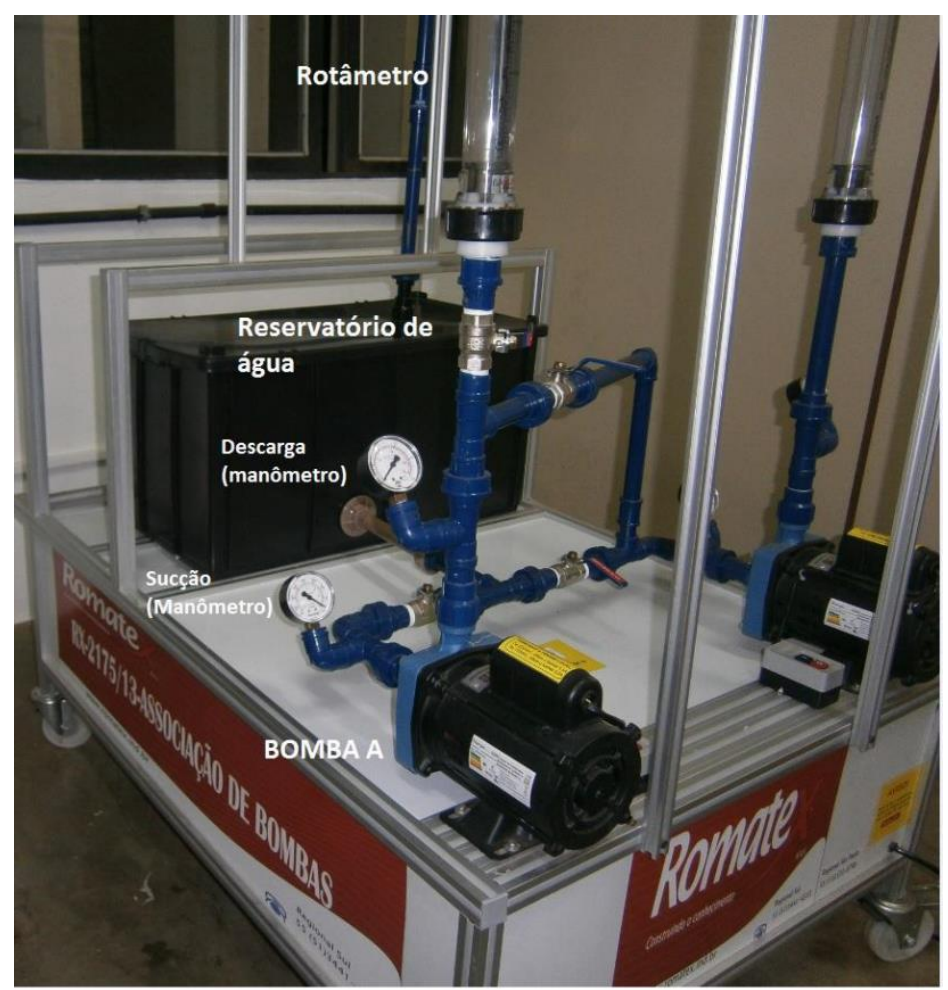

Figura 5A: Reservatório, rotâmetros, manômetros e bombas. Fonte: Autores.

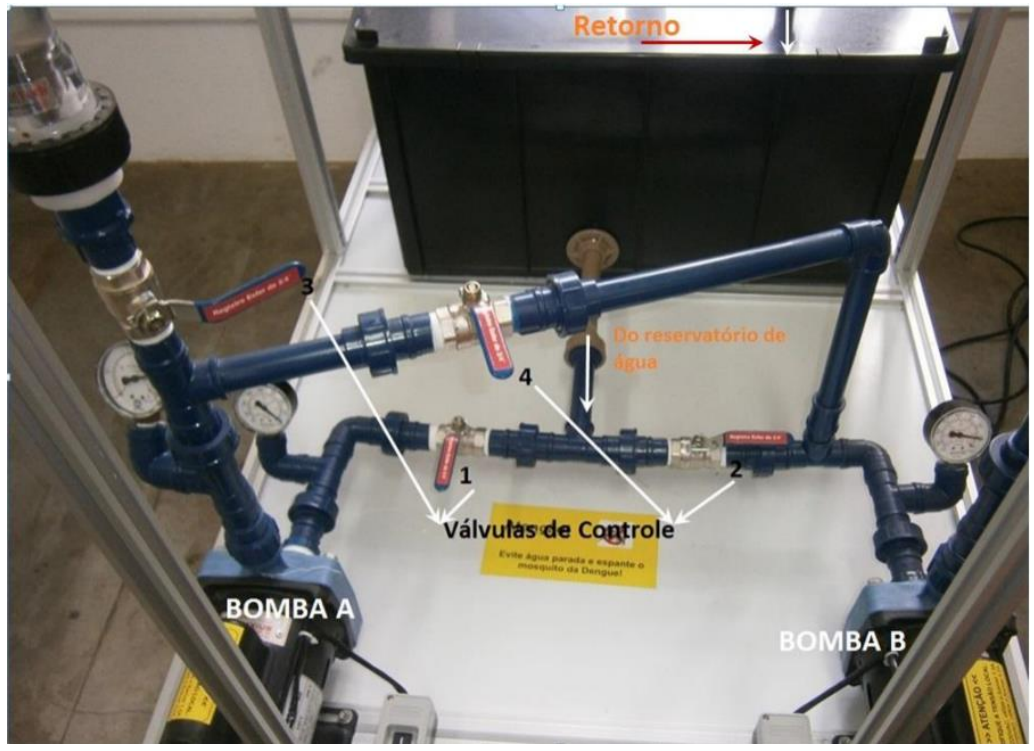

Figura 5B: Válvulas de controle. Fonte: Autores. 


\section{$\mathbf{X I X}$ CISNEMI}

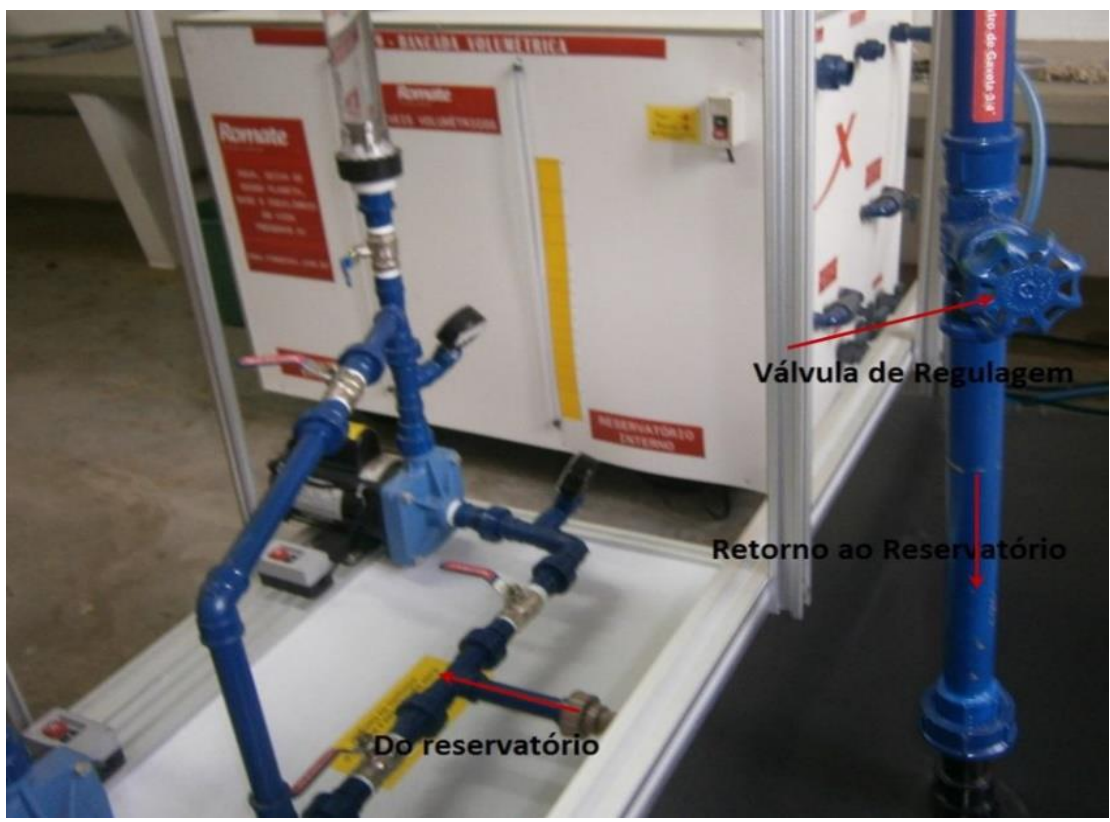

Figura 5C: Válvula de regulagem. Fonte: Autores.

A fim de se obter a curva característica da associação em série, a partir da tomada de nota dos dados a serem obtidos, estabeleceu-se a configuração de válvulas na bancada: válvulas de controle 1 e 3 abertas e válvulas 2 e 4 fechadas. Tal configuração possibilita a associação em série das bombas A e B nesta ordem. As pressões de sucção e descarga foram medidas, respectivamente, à entrada da Bomba A e à saída da Bomba B. Realizou-se a leitura de vazão no rotâmetro à saída da Bomba B. Os instrumentos lidos, bem como suas escalas e respectivas unidades, estão na Figura 6.
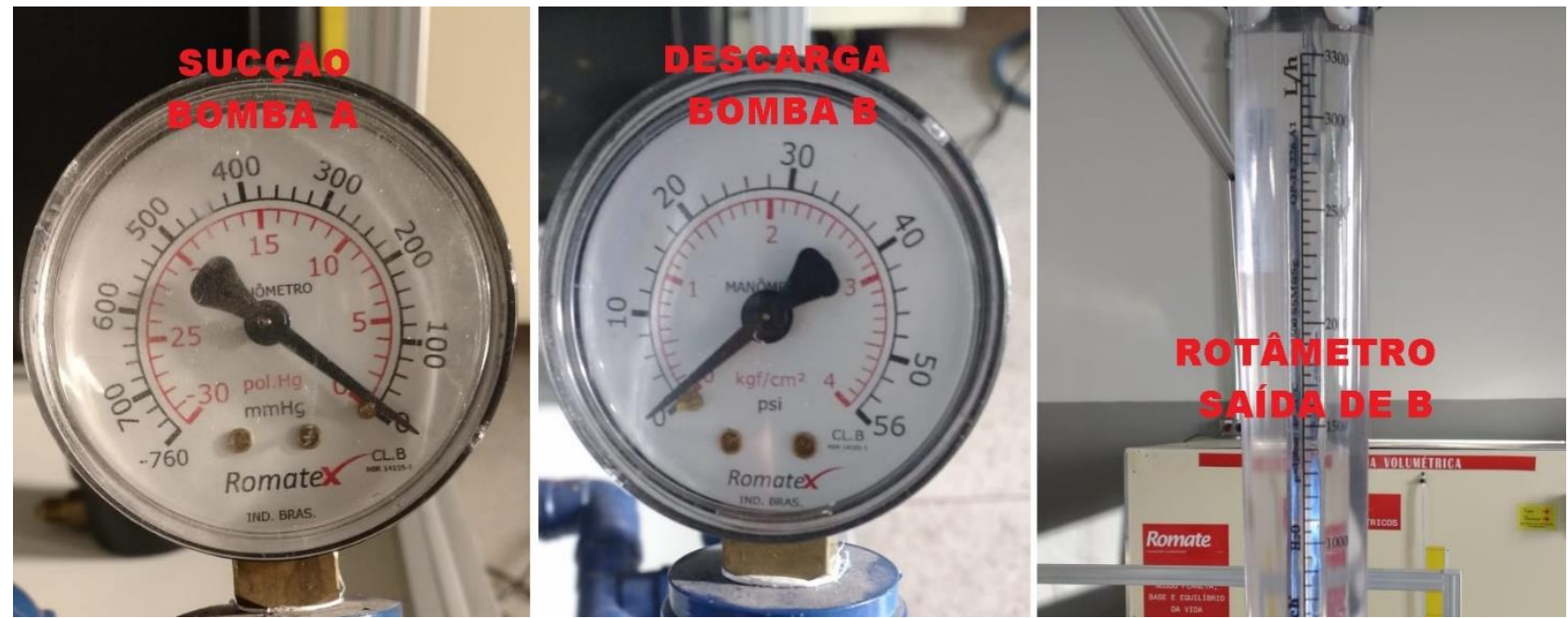

Figura 6: Instrumentos utilizados para a leitura de dados experimentais. Fonte: Autores. 


\section{$\mathbf{X I X}$ \\ CIONEMI}

A leitura inicial dos manômetros foi efetuada com a válvula de regulagem fechada, garantindo que estavam "zerados". Em seguida, após dar partida às bombas com a válvula de regulagem fechada, foram medidos os valores de pressão na sucção (entrada do sistema - anterior à primeira bomba da associação, bomba A) e na descarga (saída do sistema - posterior à segunda bomba da associação, Bomba B), assim como o valor de vazão nulo no rotâmetro à saída da Bomba B (Ponto de Bloqueio). Na sequência, a abertura da válvula de regulagem foi efetuada de forma gradual. O rotâmetro à saída da Bomba B foi o referencial para a tomada dos dados, com um intervalo de $500 \mathrm{~L} / \mathrm{h}$. Foram medidos os valores de pressão nos manômetros de sucção $(\mathrm{mmHg})$ e de descarga (psi) correspondentes para cada ponto deste intervalo até o último ponto: válvula totalmente aberta (Ponto de Operação).

\section{RESULTADOS}

Os dados experimentais obtidos são representados na Tabela 1, onde PS representa a pressão de sucção do sistema (à entrada da Bomba A) e $P D$ representam a pressão de descarga do sistema (à saída da Bomba B). RS representa a vazão no rotâmetro na saída do sistema - saída da Bomba B.

Tabela 1: Dados experimentais da associação em série. Fonte: Autores.

\begin{tabular}{|c|c|c|c|c|}
\hline Ponto & $\begin{array}{c}\text { Abertura da } \\
\text { Válvula }\end{array}$ & $\begin{array}{c}\text { PS } \\
(\mathrm{mmH})\end{array}$ & $\begin{array}{c}\text { PD } \\
\text { (Psi) }\end{array}$ & $\begin{array}{c}\text { RS } \\
(\mathrm{L} / \mathrm{h})\end{array}$ \\
\hline 1 & Fechada & 0 & 53,5 & 0 \\
\hline 2 & Aberta & 0 & 51,7 & 500 \\
\hline 3 & Aberta & 0 & 49 & 1000 \\
\hline 4 & Aberta & -20 & 46,2 & 1500 \\
\hline 5 & Aberta & $-39,9$ & 43,7 & 2000 \\
\hline 6 & Aberta & -80 & 39,6 & 2500 \\
\hline 7 & Aberta & -110 & 36,1 & 3000 \\
\hline 8 & Aberta & -158 & 32 & 3500 \\
\hline
\end{tabular}




\section{$\mathbf{X I X}$ \\ CIDNEMI}

(continuação)

\begin{tabular}{|c|c|c|c|c|}
\hline 9 & Aberta & -202 & 28,2 & 4000 \\
\hline 10 & Aberta & -270 & 24 & 4500 \\
\hline 11 & $\begin{array}{c}\text { Ponto de } \\
\text { Operação }\end{array}$ & -275 & 23,5 & 4550 \\
\hline
\end{tabular}

A Figura 7 apresenta as especificações contidas no manual do fabricante para uma família de bombas Texius. A curva característica da Bomba Centrífuga e modelo TBHWS-RN 1/2 CV é representada em cor vermelha.

APLICAÇÕES:

- Bombeamento de água limpa

- Pressurização de rede hidráulica (quando acompanhado de um sensor de acionamento automático).

- CARACTERISTICAS TÉCNICAS:

- Corpo: Polipropileno rígido

- Rotor: Nylon

- Potência: $1 / 4 \mathrm{CV}$ e $1 / 2 \mathrm{CV}$

- Tensão: 110 ou $220 \mathrm{~V}$

- Frequência: $60 \mathrm{~Hz}$

- Temperatura da água: $5^{\circ} \mathrm{C}$ a $40^{\circ} \mathrm{C}$

- Temperatura ambiente (local da instalação): $0^{\circ} \mathrm{C}$ a $40^{\circ} \mathrm{C}$

- Pressão máx. na sucção: $40 \mathrm{mca}$

- Conexões: Suc. e Rec. 1" BSP

- Peso: $6,5 \mathrm{~kg}$

- MODELOS:

- TBHWS-RN 1/4CV

- TBHWS-RN 1/2CV

- TBHWS-RD 1/2CV

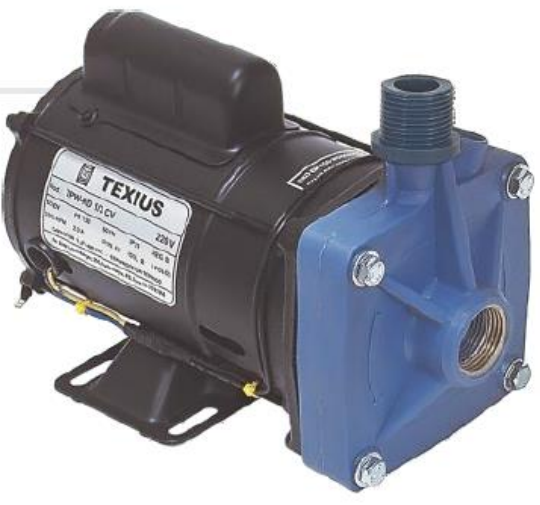

- DESEMPENHO:

Figura 7: Curvas características de bombas Texius. Fonte: Fabricante.

Comparando as unidades da curva fornecida pelo fabricante (carga de pressão em metros de coluna d'água e vazão em L/min) com as unidades dos dados experimentais, fez-se necessário aplicar os fatores de conversão de unidade: 


\section{$\mathbf{X I X}$ \\ CISNEMI}

- $1 \mathrm{~mm} \mathrm{Hg}=0,0136043233 \mathrm{MCA}$

- 1 psi $=0,7035466626 \mathrm{MCA}$

- $1 \mathrm{~L} / \mathrm{h}=0,0166666667 \mathrm{~L} / \mathrm{min}$

A Tabela 2 apresenta os dados obtidos experimentalmente com a devida conversão de unidade supracitada para fins de comparação entre os dados levantados e os contidos na curva característica fornecida pelo fabricante.

Tabela 2: Dados experimentais da associação em série - unidades corrigidas. Fonte: Autores.

\begin{tabular}{|c|c|c|c|c|}
\hline Ponto & $\begin{array}{c}\text { Abertura da } \\
\text { Válvula }\end{array}$ & $\begin{array}{c}\text { PS } \\
\text { (MCA) }\end{array}$ & $\begin{array}{c}\text { PD } \\
\text { (MCA) }\end{array}$ & $\begin{array}{c}\text { RS } \\
\text { (L/min) }\end{array}$ \\
\hline 1 & Fechada & 0 & 37,640 & 0 \\
\hline 2 & Aberta & 0 & 36,373 & 8,333 \\
\hline 3 & Aberta & 0 & 34,474 & 16,667 \\
\hline 4 & Aberta & $-0,272$ & 32,504 & 25 \\
\hline 5 & Aberta & $-0,543$ & 30,745 & 33,333 \\
\hline 6 & Aberta & $-1,088$ & 27,861 & 41,667 \\
\hline 7 & Aberta & $-1,497$ & 25,398 & 50 \\
\hline 8 & Aberta & $-2,150$ & 22,514 & 58,333 \\
\hline 9 & Aberta & $-2,748$ & 19,840 & 66,667 \\
\hline 10 & Aberta & $-3,673$ & 16,885 & 75 \\
\hline 11 & $\begin{array}{c}\text { Ponto de } \\
\text { Operação }\end{array}$ & $-3,741$ & 16,533 & 75,833 \\
\hline
\end{tabular}

A carga que uma bomba centrífuga entrega ao fluído é medida através da diferença entre pressão de descarga e pressão de sucção; para um sistema associado em série, idem: subtração entre pressão de descarga (saída da última bomba) e de sucção (entrada da primeira bomba). A carga entregue ao fluido pela associação em série, para cada uma das vazões dadas em cada ponto amostrado no experimento, é apresentada abaixo na Tabela 3. 


\section{$\mathbf{X I X}$ \\ CINEMI}

Tabela 3: Dados experimentais da associação em série: carga vs vazão. Fonte: Autores.

\begin{tabular}{|c|c|c|c|}
\hline Ponto & $\begin{array}{c}\text { Abertura da } \\
\text { Válvula }\end{array}$ & $\begin{array}{c}\text { Carga } \\
\text { (MCA) }\end{array}$ & $\begin{array}{c}\text { Vazão } \\
\text { (L/min) }\end{array}$ \\
\hline 1 & Fechada & 37,640 & 0 \\
\hline 2 & Aberta & 36,373 & 8,333 \\
\hline 3 & Aberta & 34,474 & 16,667 \\
\hline 4 & Aberta & 32,776 & 25 \\
\hline 5 & Aberta & 31,288 & 33,333 \\
\hline 6 & Aberta & 28,949 & 41,667 \\
\hline 7 & Aberta & 26,896 & 50 \\
\hline 8 & Aberta & 24,663 & 58,333 \\
\hline 9 & Aberta & 22,589 & 66,667 \\
\hline 10 & Aberta & 20,558 & 75 \\
\hline 11 & $\begin{array}{l}\text { Ponto de } \\
\text { Operação }\end{array}$ & 20,275 & 75,833 \\
\hline
\end{tabular}

Observou-se que a vazão máxima do sistema (ponto de operação) apresentou um valor aproximado de $76 \mathrm{~L} / \mathrm{min}$. Portanto, optou-se por amostrar os pontos de carga, no gráfico fornecido pelo fabricante, até um máximo de vazão equivalente (com intervalo de $5 \mathrm{~L} / \mathrm{min}$ ). A Tabela 4 , a seguir, contém os dados de carga por vazão, tomados na curva fornecida pelo fabricante, para cada ponto definido. A leitura dos pontos foi efetuada a olho nu no manual do fabricante que contém a curva característica apresentada anteriormente na Figura 7. 


\section{$\mathbf{X I X}$ \\ CIDNEMI}

Tabela 4: Dados do fabricante: carga vs vazão. Fonte: Autores.

\begin{tabular}{|c|c|c|}
\hline Ponto & Carga (MCA) & $\begin{array}{c}\text { Vazão } \\
\text { (L/min) }\end{array}$ \\
\hline 1 & 17 & 0 \\
\hline 2 & 16,8 & 5 \\
\hline 3 & 16,3 & 10 \\
\hline 4 & 16 & 15 \\
\hline 5 & 15,9 & 20 \\
\hline 6 & 15,5 & 25 \\
\hline 7 & 15 & 30 \\
\hline 8 & 14,5 & 35 \\
\hline 9 & 14,1 & 40 \\
\hline 10 & 13,6 & 45 \\
\hline 11 & 13,2 & 50 \\
\hline 12 & 12,5 & 55 \\
\hline 13 & 12 & 60 \\
\hline 14 & 11,2 & 65 \\
\hline 15 & 10,2 & 70 \\
\hline 16 & 9,6 & 75 \\
\hline 17 & 9,4 & 76 \\
\hline
\end{tabular}

Através dos dados da Tabela 4 (dados da curva do fabricante), obteve-se a curva característica teórica da associação em série, para cada um dos pontos. O valor da carga resultante na associação é o dobro do valor de carga de uma única bomba, para uma mesma vazão; uma vez que se tratam de bombas idênticas - conforme mostrado anteriormente na Figura 3. O Gráfico 1, construído no Excel, apresenta a curva característica teórica para a associação em série representada pela linha de tendência, obtida através dos pontos amostrados na curva do fabricante. 


\section{XIX \\ CONEMI}

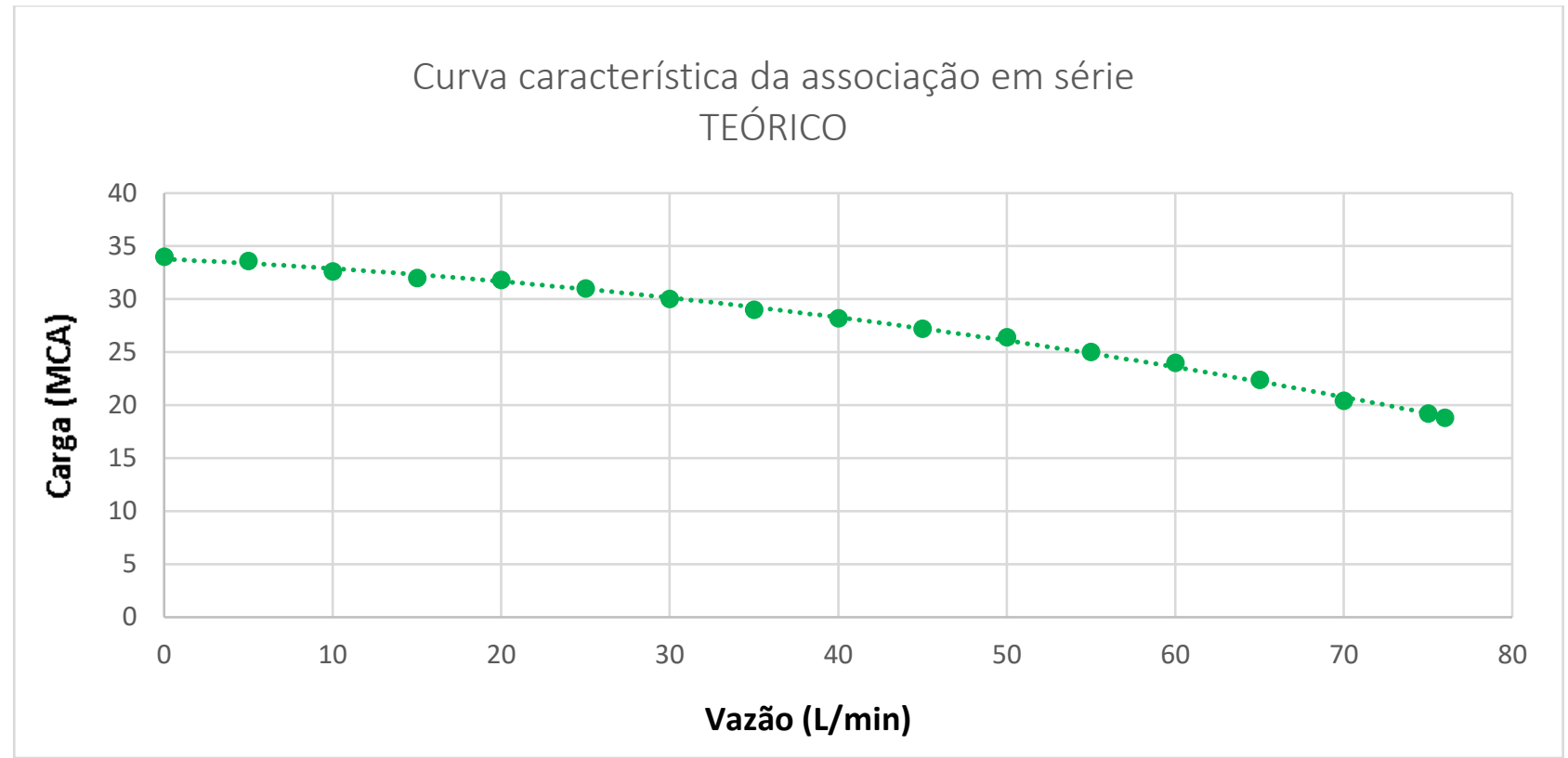

Gráfico 1: Curva característica teórica da associação em série. Fonte: Autores.

Através da Tabela 3 (dados experimentais), construímos o Gráfico 2 - idem, via Excel. Tal gráfico representa o comportamento experimental do sistema constituído pelas bombas A e B quando associadas em série. A função correspondente à linha de tendência é apresentada (função polinomial de segundo grau, típica para o comportamento de bombas centrífugas); onde " $y$ " é o valor de carga e " $x$ " o de vazão. O valor de R à segunda potência (coeficiente de determinação medida de ajustamento de um modelo estatístico linear) próximo ao de uma unidade aponta uma boa aproximação (representação) dos dados. Mais especificamente, o valor encontrado de $\mathrm{R}$ à segunda potência, para os dados especificados, aponta uma precisão de aproximadamente 99,93\% para a função associada à linha de tendência quanto à representação dos dados. Esta precisão, porém, refere-se exclusivamente à representatividade dos pontos amostrados pela função associada á linha de tendência. Erros de amostragem experimental não são considerados neste momento e são discutidos posteriormente via análise de incerteza experimental. 


\section{$\mathrm{XIX}$ \\ CISNEMI}

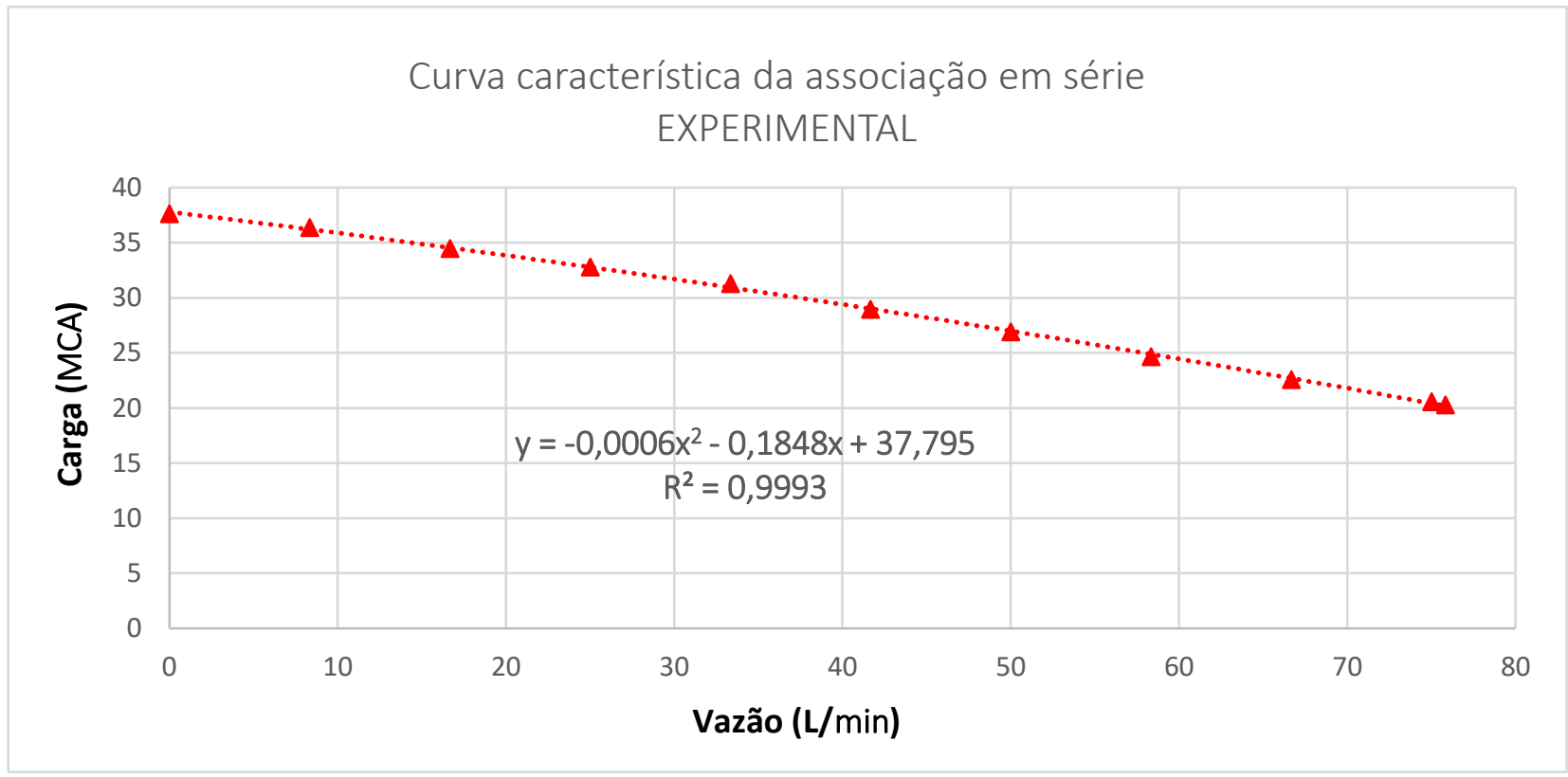

Gráfico 2: Curva característica experimental da associação em série. Fonte: Autores.

O Gráfico 3 apresenta a sobreposição das curvas experimental (vermelha) e teórica (verde) para a associação em série.

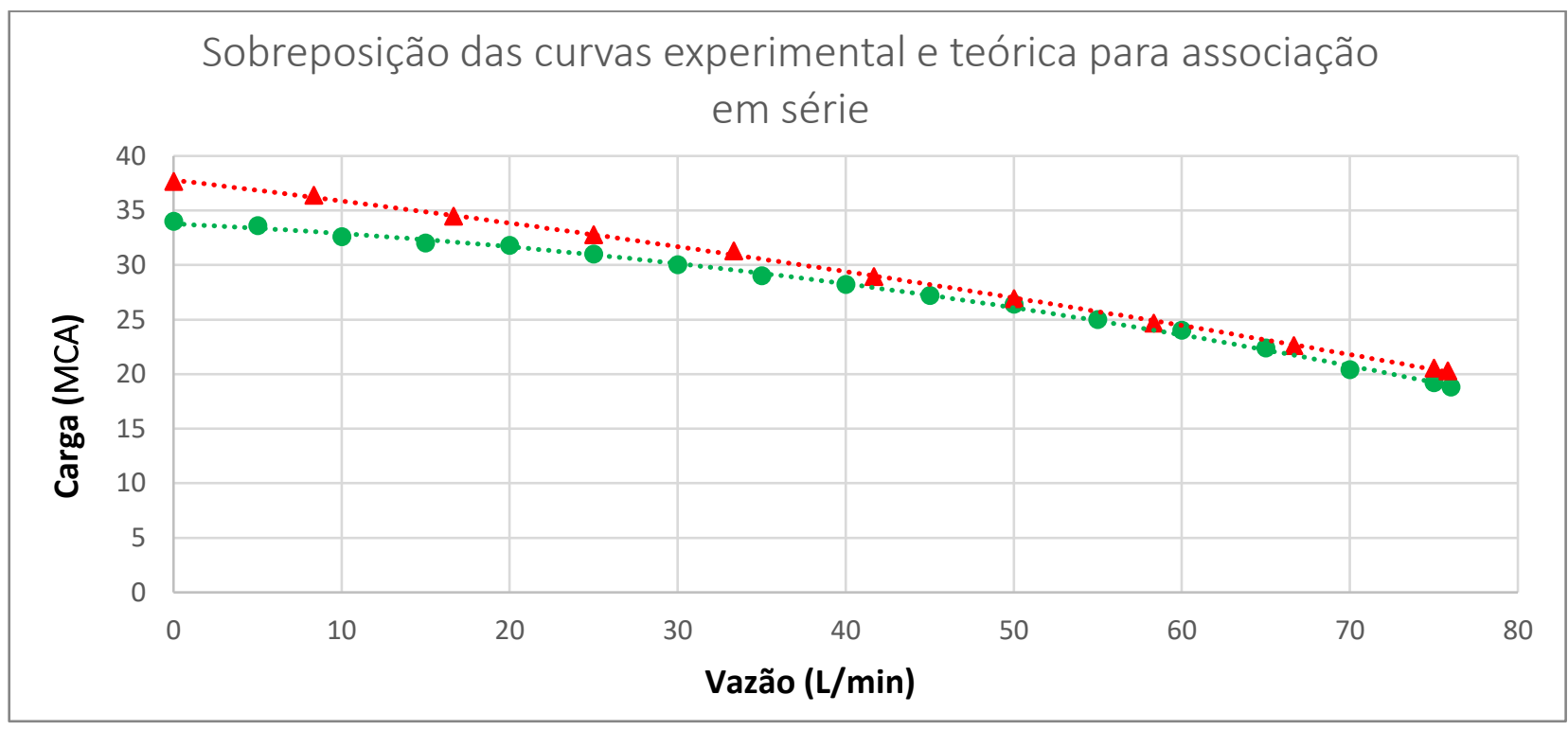

Gráfico 3: Sobreposição das curvas experimental e teórica da associação em série. Fonte: Autores. 


\section{$\mathbf{X I X}$ \\ CIONEMI}

\section{ANÁLISE DE RESULTADOS E CONCLUSÕES}

Seguindo a mesma metodologia proposta por SILVA (2018), porém dessa vez para efetuar - levantamento da curva característica para a associação das bombas de estudo em série, a proximidade entre a curva experimental da associação quando comparada à curva obtida de forma teórica mostrou-se igualmente adequada e suficiente (Gráfico 3). De praxe, como no trabalho de SILVA (2018), o pequeno desvio apresentado entre os diferentes métodos pode ser explicado pela presença de erros comuns associados à leitura dos instrumentos analógicos, durante o procedimento experimental, que é efetuada a olho nu; bem como erros associados às calibrações dos mesmos. Cabe, portanto, análise quanto à incerteza experimental.

Conforme Figura 6 (página 8), o manômetro na sucção da Bomba A apresenta escala com divisão de 20 mmHg; o manômetro na descarga da Bomba B apresenta escala com divisão de 2 psi; e o rotâmetro à saída de B apresenta escala com divisão de $50 \mathrm{~L} / \mathrm{h}$. Assumindo um possível erro de leitura a olho nu e de calibração dos instrumentos de \pm o valor da divisão da escala para cada instrumento e aplicando os fatores de conversão de unidade listados na página 10, temos:

- Erro no manômetro de sucção da Bomba $\mathrm{A}= \pm 20 \mathrm{mmHg}= \pm 0,272 \mathrm{MCA}$

- Erro no manômetro de descarga da Bomba $B= \pm 2 p s i= \pm 1,407$ MCA

- Erro no rotâmetro à saída da Bomba $\mathrm{B}= \pm 50 \mathrm{~L} / \mathrm{h}= \pm 0,833 \mathrm{~L} / \mathrm{min}$

O erro no valor da carga para cada ponto amostrado é a soma entre o erro associado aos manômetros de sucção e descarga e o erro associado à leitura de vazão. O erro no valor da carga associado às leituras de sucção e descarga pode ser obtido através da diferença entre os erros presentas na descarga e na sucção, respectivamente. A função erro no valor da carga associado à leitura de vazão para cada ponto pode ser definida ao subtrair a linha de tendência obtida no Gráfico 2 da função obtida ao substituir a variação do erro do rotâmetro na mesma linha de tendência.

- Erro no valor carga associado aos instrumentos de sucção e descarga = $( \pm 1,407)-( \pm 0,272)= \pm 1,679$ 


\section{$\mathbf{X I X}$ \\ CIONEMI}

- Erro no valor da carga associado à leitura de vazão = $-0,0006 .(\mathrm{x} \pm 0,833)^{2}-0,1848 .(\mathrm{x} \pm 0,833)+37,795-\left[-0,0006 .(\mathrm{x})^{2}-\right.$ $0,1848 .(\mathrm{x})+37,795]=0,0006 \cdot(\mathrm{x})^{2}+0,1848 .(\mathrm{x})-0,0006 \cdot(\mathrm{x} \pm 0,833)^{2}-$ $0,1848 .(x \pm 0,833)$ $\cong \pm 0,0009996 .(x+154,0005) \cong \pm(0,001 x+0,154)$

- Erro no valor da carga para cada ponto $\cong$ $\pm 1,679 \pm(0,001 x+0,154) \cong \pm 1.833 \pm 0,001 x$

A Tabela 5 representa o intervalo de erro para cada ponto amostrado experimentalmente.

Tabela 5: Erro para cada ponto amostrado experimentalmente. Fonte: Autores.

\begin{tabular}{|c|c|c|}
\hline Ponto & $\begin{array}{l}\text { Vazão } \\
\text { (L/min) }\end{array}$ & $\begin{array}{l}\text { Erro } \\
\text { MCA }\end{array}$ \\
\hline 1 & 0 & $\pm 1,833$ \\
\hline 2 & 8,333 & $\pm 1,841$ \\
\hline 3 & 16,667 & $\pm 1,850$ \\
\hline 4 & 25 & $\pm 1,858$ \\
\hline 5 & 33,333 & $\pm 1,866$ \\
\hline 6 & 41,667 & $\pm 1,875$ \\
\hline 7 & 50 & $\pm 1,883$ \\
\hline 8 & 58,333 & $\pm 1,891$ \\
\hline 9 & 66,667 & $\pm 1,900$ \\
\hline 10 & 75 & $\pm 1,908$ \\
\hline 11 & 75,833 & $\pm 1,909$ \\
\hline
\end{tabular}

Na sequência abaixo, apresenta-se o Gráfico 4, análogo ao Gráfico 3; contendo, porém, a variação do erro na curva experimental para cada ponto amostrado. Nota-se que a diferença entre as curvas experimental e teórica é significativamente atenuada, uma vez que a curva teórica se aproxima das tolerâncias para cada valor experimental. No entanto, para baixas vazões ainda há 


\section{XIX \\ CIONEMI}

uma distância significativa entre as duas curvas; fato justificável através de uma simples análise dos dados levantados experimentalmente, presentes na Tabela 1, página 8: observa-se que para os três primeiros pontos medidos, a pressão de sucção apresentou-se fixa em valor nulo - o que só deveria acontecer para o ponto de bloqueio (vazão nula). Isso se deve, exclusivamente, à sensibilidade do manômetro presente na sucção da Bomba A, que não se apresentou suficiente para detectar as variações na pressão de sucção para baixas vazões - mesmo quando considerado um erro de leitura de \pm o valor da menor escala do instrumento. Tal manômetro, portanto, necessita de substituição para aquisição mais precisa dos dados. Outros erros presentes, porém, minimamente significantes, são a amostragem a olho nu de pontos na curva do fabricante e a aproximação dos dados por linha de tendência.

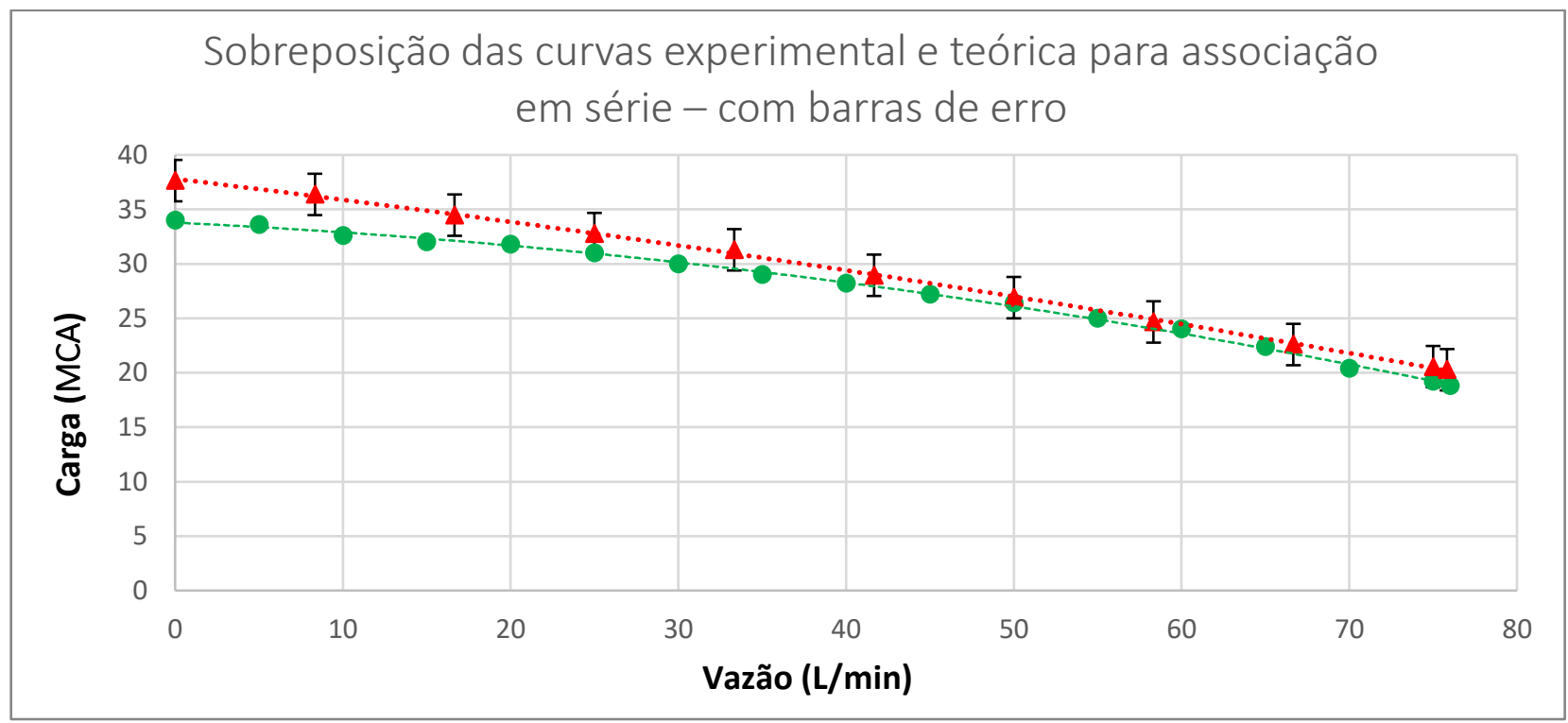

Gráfico 4: Curvas experimental (com barras de erro) e teórica da associação em série. Fonte: Autores.

Outra observação cabível para análise de resultado refere-se ao rendimento das bombas, assim como feito por SILVA (2018). Segundo Selo Procel de Economia de Energia das bombas, são dados de operação para o ponto de máximo rendimento: Vazão $=5,33 \mathrm{~m}^{3} / \mathrm{h}=88,83 \mathrm{~L} / \mathrm{min}$ e Carga = 12,2 MCA. A vazão máxima do sistema para associação em série foi de aproximadamente 76 L/min. Esta é também a vazão em cada bomba, já que na associação em série as vazões das bombas 


\section{$\mathbf{X I X}$ \\ CISNEMI}

são iguais entre si, cujo valor é igual à vazão do sistema. Este valor se apresenta razoavelmente próximo à vazão para ponto de máximo rendimento; diferente da associação em paralelo, apresentada por SILVA (2018), onde apesar de o sistema apresentar uma vazão resultante de aproximadamente $80 \mathrm{~L} / \mathrm{min}$, cada bomba, individualmente, possui a vazão aproximada de $40 \mathrm{~L} / \mathrm{min}$.

O Gráfico 5 apresenta a sobreposição das curvas experimentais para a associação em série (vermelha) obtida no presente trabalho e para a associação em paralelo (preta) obtida por SILVA (2018). Tal sobreposição possibilita a mensuração visual das características inerentes a cada tipo de associação (maior carga para a associação em série e maior vazão para a associação em paralelo). Torna-se evidente, inclusive, a superioridade da associação em série para este sistema em específico. Afinal, para o ponto de operação - válvula totalmente aberta -, a associação em série, além de apresentar maior carga (aproximadamente $20 \mathrm{MCA}$ ), possui cada bomba operando com vazão razoavelmente próxima à de máximo rendimento (como apontado no parágrafo anterior). 0 ganho de vazão no ponto de operação ao utilizar a associação em paralelo é mínimo (aproximadamente $4 \mathrm{~L} / \mathrm{min}$ ), ao custo de cada uma das bombas operarem com valores de vazão muito abaixo (aproximadamente metade) da correspondente ao máximo rendimento $(88,33 \mathrm{~L} / \mathrm{min})$.

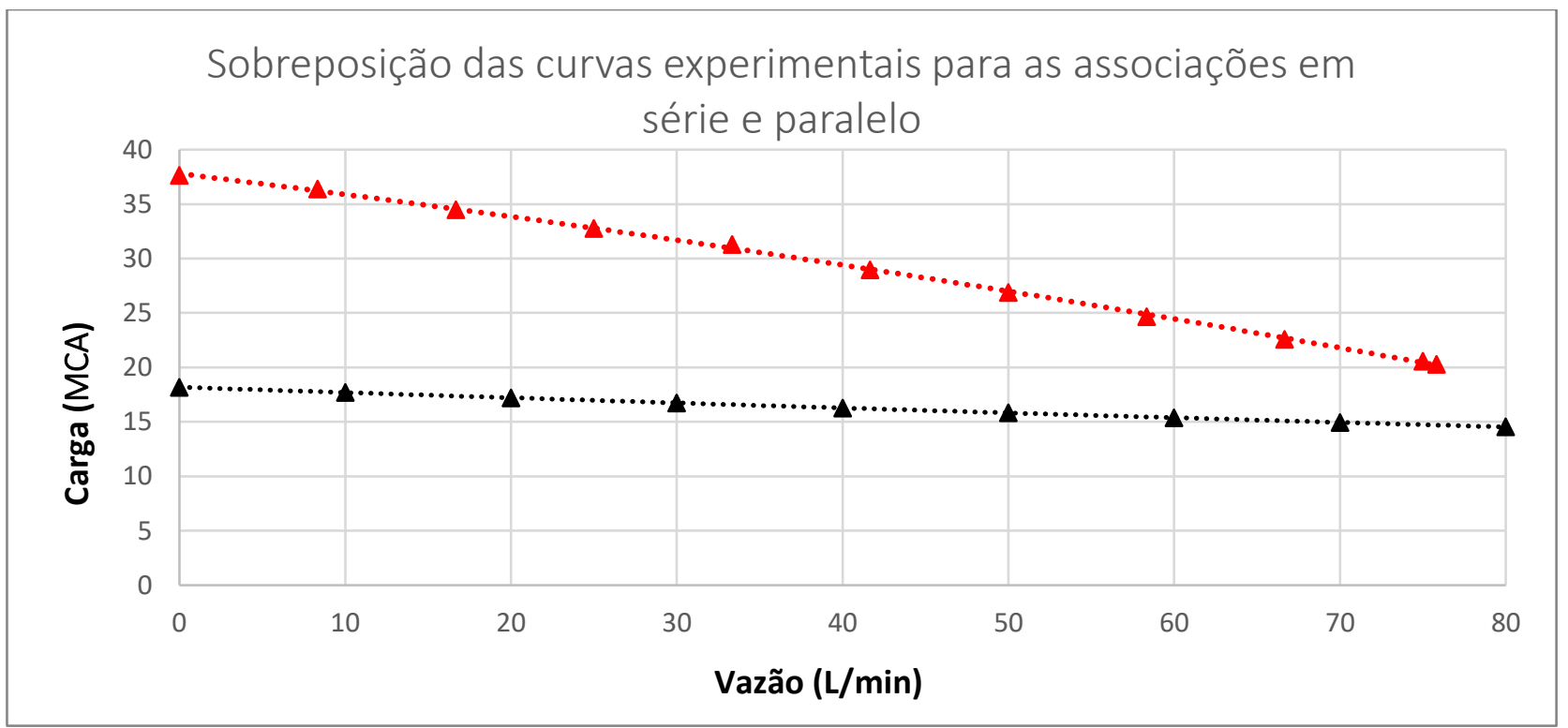

Gráfico 5: Sobreposição das curvas experimentais para ambas associações. Fonte: Autores. 


\section{$\mathbf{X I X}$ \\ CISNEMI}

\section{REFERÊNCIAS}

SILVA, R.J. LEVANTAMENTO EXPERIMENTAL DA ASSOCIAÇÃO EM PARALELO DE BOMBAS CENTRÍFUGAS TEXIUS MODELO TBHWS-RN $1 \frac{1}{2}$ CV. In: ANAIS DO XVIII CONGRESSO NACIONAL DE ENGENHARIA MECâNICA E INDUSTRIAL, 2018, Joinville. Anais eletrônicos... Campinas, GALOÁ, 2018. Disponível em: <https://proceedings.science/anais-do-conemi/conemi-2018/papers/levantamento-experimental-da--associacao-em-paralelo-de-bombas-centrifugas-texius-modelo-tbhws-rn-

\%C2\%BD-cv> Acesso em: 2019.

FOX R.W, MCDONALD A e PRITCHARD PJ. Introdução à Mecânica dos Fluidos. Ed. Guanabara Koogan, 7ạ edição. LTC Editora (2001);

WHITE FM. Fluid Mechanics. McGraw Hill, 6 edition, p. 763-825, 2011;

MACINTYRE, A. J. Bombas e instalações de bombeamento. LTC. 1987;

CARVALHO D.F. Instalações Elevatorias. Bombas, 6ạ edição, p. 1-159.

MACINTYRE, A.J., Bombas e instalações de bombeamento. Rio de Janeiro, Editora Guanabara, 1982. Especificações da Bomba Centrífuga TEXIUS, modelo TBHWS-RN 1⁄2 CV. 